Markus Behmer/ Jeffrey Wimmer

\section{Mehr Schein als Sein?}

Internationale und interkulturelle Kommunikation als Themen kommunikationswissenschaftlicher Lehre

\title{
Die Ausgangslage
}

$\mathrm{Zu}$ Beginn des neuen Jahrtausends wurde bei einer Hamburger Tagung Resümee gezogen, inwieweit sich die deutschsprachige Kommunikationswissenschaft bisher mit dem Forschungsbereich „internationale und interkulturelle Kommunikation" auseinandergesetzt hat. Die im Band „Zukunft der internationalen Kommunikationswissenschaft" versammelten Stimmen kommen zu einem enttäuschten Zwischenfazit (Hafez 2002a). Für sie verharrte die deutsche Kommunikationswissenschaft bei einem eng nationalen Fokus, internationale Themen stünden nicht hoch auf der Agenda. Kai Hafez (2002b, S.9) spitzte diese Diagnose zu: „Die internationale Kommunikations- und Medienwissenschaft in Deutschland muss in Zukunft erhebliche Anstrengungen unternehmen, um im Weltmaßstab konkurrenzfähig zu werden.“ Kunczik (2002) resümierte, dass wir nicht nur ein „falsches Bild" in der Berichterstattung über andere Länder feststellen könnten sondern auch in den Köpfen der Menschen; eine verzerrte Wahrnehmung, von der auch die Kommunikationsexperten unserer Mediengesellschaft nicht gefeit seien - wie die Beschreibungen des niederländischen Journalisten Joris Luyendijk (2007) aus dem Alltag der Nahost-Krisenberichterstattung exemplarisch verdeutlichen.

Wenigstens im Feld international vergleichender Analysen tut sich aber zuletzt Einiges. So sprachen Frank Esser und Barbara Pfetsch bereits vor fünf Jahren sogar von einer „rasante(n) Entwicklung der einschlägigen Forschung“: „In den 1990er Jahren haben sich produktive Netzwerke von international arbeitenden Forscherinnen und Forscher gebildet, die eine Reihe markanter Projekte auf den Weg gebracht haben. Und der Prozess der Europäisierung verleiht den Aktivitäten diesseits des Atlantik nochmals neuen Impetus" (Esser/ Pfetsch 2003b, S. 10). Vergleichende Forschung zumindest zur politischen Kommunikation sei international, so Michael Gurevitsch und Jay G. Blumler (2003, S. 372f.), ja "fast schon in Mode gekommen!“ Das in ihr liegende Potential sei „erkannt und die Wertschätzung und die Anwendung vergleichender Ansätze“ habe sich "durchgesetzt“. Begründet sehen sie dies unter anderem in der vor allem durch neue Kommunikationstechniken ausgelösten Globalisierung, die auch zu 
einer Homogenisierung der politischen Kommunikation über politische und kulturelle Grenzen hinweg führen könne, aber auch ganz pragmatisch in verbesserten Reise- und Kommunikationsmöglichkeiten unter Forschern und im einfacheren Zugang zu Informationen und Daten, die gemeinsame Forschungsprojekte umstandsloser machten. Und Sonia Livingstone (2003, S. 477) weißt weiter darauf hin, dass auch Geldgeber und politische Vorgaben komparative Forschung zunehmend begünstigten.

So zeigt dann auch eine von Josef Seethaler (2006) vorgenommene Meta-Analyse von vier führenden europäischen kommunikationswissenschaftlichen Fachzeitschriften auf, dass die Zahl der Beiträge mit europäisch-vergleichender Perspektive seit 1998 deutlich angestiegen ist (wiewohl sie am Ende des Untersuchungszeitraums immer noch weniger als zehn Prozent aller Beiträge ausmacht). Eine von Thomas Hanitzsch und Klaus-Dieter Altmeppen (2007) unternommene Langzeitanalyse der Publikationen mit vergleichendem Fokus in drei deutschen Fachzeitschriften über fast 60 Jahre hinweg zeigt ebenso eine deutliche Zunahme komparativer Forschung seit Mitte der neunziger Jahre.

Deutschland ist zwar weiterhin keinesfalls führend in diesem sich dynamisch entwickelnden Forschungsfeld, doch wurde in den vergangenen Jahren immerhin eine Reihe von Fachtagungen mit internationalen Themenfeldern in Deutschland veranstaltet und auch die Präsenz deutscher Forscherinnen und Forscher auf internationalen Tagungen und in englischsprachigen Fachzeitschriften hat stark zugenommen. Die Broschüre „How to go international“ (Bilandzic et al. 2004) der Deutschen Gesellschaft für Publizistik- und Kommunikationswissenschaft zeigt den institutionellen Rahmen und das Anliegen der DGPuK zur Förderung. Über die Qualität der Forschung ist damit freilich noch nichts ausgesagt. Doch scheint die von Hans J. Kleinsteuber (2002, S. 43) ebenfalls auf der Hamburger Tagung formulierte Forderung, „ein Deutschland, das sich in einer rasch wandelnden und zunehmend globalisierten Medienlandschaft orientieren und identifizieren will, wird die bisher gepflegte nationale Nabelschau rasch aufgeben müssen“, zunehmend umgesetzt zu werden. Es gibt - auch hier - einen Trend zu internationaler Forschung; eine Sensibilisierung für den Themenbereich zeichnet sich ab.

Dennoch hat sich auch die Kritik vermehrt. Am prominentesten hat in letzter Zeit der Wissenschaftsrat der Kommunikations- und Medienwissenschaft in Deutschland ein schlechtes Zeugnis ausgestellt (2007). So konstatiert er unter anderem eine fehlende internationale Wahrnehmung der deutschen Forschung und einen fehlenden Willen, in der Forschung international anschlussfähig zu sein (S. 98f.). 
In eine ähnliche Richtung geht eine finnische Analyse der deutschen Kommunikationswissenschaft, die unter Bezugnahme auf eine Analyse der Rezensionen in der „Publizistik“ schlussfolgert, dass gerade im Vergleich zu den europäischen Nachbarn die fehlende internationale Ausrichtung in Deutschland symptomatisch sei (vgl. Koivisto/ Thomas 2007, S. 63).

Der Wissenschaftsrat beließ es in seinem Memorandum nicht bei der Kritik, sondern gab im Hinblick etwa auf die Ausrichtung der Lehre drei - eher allgemein gehaltene - Empfehlungen (Wissenschaftsrat 2007, S. 99): Die Studierenden sollten verstärkt durch Auslandstipendien gefördert, Seminarangebote vermehrt mehrsprachig - in der Regel englisch - gehalten und ausländische Gastdozenten zunehmend in den Lehrbetrieb integriert werden. Fehlende internationale Ausrichtung in der Ausbildung hatte Hans J. Kleinsteuber ebenfalls bereits 2002 festgehalten. „Weder ist sie fest in den Kanon der kommunikationswissenschaftlichen Lehre eingebunden, noch gibt es einführende Standardwerke" (Kleinsteuber 2002, S. 42).

Wenigstens in Bezug auf das zweite Monitum hat sich mittlerweile Einiges getan. Einen Eckpunkt markierten Barbara Pfetsch und Frank Esser, die 2003 ein international angelegtes Überblickswerk zur „Politische(n) Kommunikation im internationalen Vergleich“ vorlegten, das auch in einer englischen Ausgabe erschien. 2007 legte Barbara Thomaß einen als Lehrbuch konzipierten Sammelband über „Mediensysteme im internationalen Vergleich“ vor. 2008 veröffentlichten Gabriele Melischek, Josef Seethaler und Jürgen Wilke einen umfassenden Reader zu „Medien und Kommunikationsforschung im Vergleich“. Und mit einer zum Teil an den Cultural Studies orientierten Perspektive veröffentlichte insbesondere Andreas Hepp in jüngster Zeit gleich mehrere Titel. So edierte er gemeinsam mit Martin Löffelholz 2002 einen Sammelband mit „Grundlagentexte(n) zur Transkulturellen Kommunikation“, 2005 gab er zusammen mit Friedrich Krotz und Carsten Winter ein Einführungsbuch zur „Globalisierung der Medienkommunikation“ heraus, 2006 verfasste er ein Lehrbuch „Transkulturelle Kommunikation“.

Die Rahmenbedingungen auch für die Lehre im Hinblick auf die Vermittlung internationaler und interkultureller Themen und Problemstellungen scheinen also weit günstiger als noch vor einigen Jahren. In unserem Beitrag suchen wir nun Indikatoren, ob das entstandene Framework genutzt wird - oder ob die zum Teil harschen Urteile auch heute noch angebracht sind. Unsere zentrale Frage lautet daher: Inwieweit ist das Lehrangebot der Kommunikations- und Medienwissenschaft „internationalisiert“? 
Ausgehend vom Humbold'schen Ideal der Einheit von Forschung und Lehre ist danach $\mathrm{zu}$ fragen, inwieweit aktuelle Prozesse der Transnationalisierung und Globalisierung mit eigenständigen Kursen - und nicht nur in der Forschung - reflektiert werden. Lehre in diesem Bereich erscheint uns zunehmend wichtig, da die Globalisierung der Medienkommunikation auch zu einer doppelten Anforderung der Studierenden der Kommunikations- und Medienwissenschaft führt: Sie werden einerseits in ihrem Alltag damit konfrontiert, u. a. mit einer zunehmend multikulturelleren Gesellschaft, andererseits müssen sie im späteren Berufsleben z. B. als Journalist vermehrt mit den Anforderungen und Spezifika einer globalisierten und transnationalen Medienkommunikation zurechtkommen und im Idealfall gestaltend Einfluss nehmen können. Gerade im Bereich der Journalistenausbildung wurde daher frühzeitig erkannt und eingefordert, dass die Multikulturalität der Gesellschaft in den Curricula und in spezifischen Lehrinhalten sowohl reflektiert als auch repräsentiert wird (z. B. Baldasty et al. 2003); darüber hinaus sollte die zunehmende Globalisierung der Medienkommunikation auch in einer Internationalisierung der Journalistenausbildung ihren Widerhall finden.

Inwieweit die skizzierten Postulate und Prozesse nun in der Lehre an deutschen Universitäten und Fachhochschulen eingelöst sind, wollen wir durch einen inhaltsanalytischen, quantifizierenden Blick auf die verschiedenen, aktuell angebotenen Vorlesungen, Übungen und Seminarangebote prüfen. Ein Beispiel aus der universitären Praxis, eine Universität und Alltagshandeln, Forschung und Anwendungsorientierung verbindende Seminarreihe ${ }^{1}$, soll dann exemplarisch Möglichkeiten der Lehre im Bereich der internationalen und interkulturellen Kommunikation verdeutlichen. Vorläufige Trendbeobachtungen und ein Ausblick schließen den Beitrag ab.

\section{Kursangebote im Bereich „Internationale Kommunikation"}

Die Schaffung eines gemeinsamen europäischen Bildungsraumes wird immer wieder als ein zentrales Anliegen der EU-Kultuspolitik beschworen. Der deutlichste Schritt zur Umsetzung dieses Vorhabens ist die „Internationalisierung“ der Studienabschlüsse im Bologna-

1 Die Tagungsreihe gestalten die Autoren gemeinsam mit der Internationalen Weiterbildung und Entwicklung gGmbh, der Deutschen Journalistenschule und dem Internationalen Institut für Journalismus sowie dem Verein Nachwuchsjournalisten in Bayern und seit 2007 auch der Ausbildungsredaktion des Bayerischen Rundfunks (vgl. Kapitel Seminarreihe „Bildkorrekturen“). 
Prozess. Zumindest formal ergibt sich eine leichtere Anrechenbarkeit von im Ausland erbrachten Studienleistungen über das ECTS-System. Auch die steigende Zahl von Erasmus-Partnerschaften in Europa verdeutlicht die zunehmende Internationalisierung. ${ }^{2}$ Weitere internationale Bestrebungen zur Anhebung des allgemeinen Standards der Lehre gibt es gerade im Bereich der Entwicklungskommunikation - so hat die Unesco 2007 einen Modelllehrplan für Journalistenausbildung in „developing countries and emerging democracies" aufgelegt.

Trotz des oben skizzierten günstigen allgemeinen Frameworks gibt es in der deutschen Medien- und Kommunikationswissenschaft bislang nur wenige (und zum Teil sehr kleine) dezidiert internationale Studiengänge - wie exemplarisch den „Euro Mundus Master: Journalism and Media within Globalisation", den die Universität Hamburg zusammen mit vier europäischen Partneruniversitäten durchführt, den "Master in International Communication“ an der Jacobs University Bremen oder den zum Wintersemester 2008/09 gestarteten Masterstudiengang „International Media Studies“, den die Universität Bonn gemeinsam mit der Deutschen Welle und der Fachhochschule Bonn Rhein-Sieg anbietet. Ferner gibt es einige binationale, speziell deutsch-französische Studiengänge - so die Masterprogramme „Europäische Medienkultur" und „Medienmanagement", die beide von der Bauhaus-Universität Weimar gemeinsam mit der Université Lumière Lyon 2 offeriert werden (ersterer bereits seit 1998), das Masterprogramm „Deutsch-Französische Journalistik/Master professionnel du journalisme", das vom Frankreich-Zentrum der Universität Freiburg ebenfalls zusammen mit der Universität Lyon 22005 ins Leben gerufen wurde, und „Europajournalismus und europäische Öffentlichkeit“, gleichfalls ein Masterprogramm, das von der RWTH Aachen gemeinsam mit der Université Reims seit dem Herbst 2008 betrieben wird. Trinational organisiert ist der Master „Medien - Kommunikation Kultur", bei dem die Viadrina Universität Frankfurt/Oder mit der französischen Université de Nice Sophia Antipolis und der bulgarischen St. Kliment Ohridski Universität Sofia kooperiert.

Ganze fünf Professuren mit spezifisch internationaler oder interkultureller Widmung sind an kommunikationswissenschaftlichen In-

2 So nutzen im akademischen Jahr 2006/07 fast 160000 Studierende der 31 heute am (seit 1987 bestehenden) Erasmus-Programm der EU partizipierenden Staaten die Möglichkeit, ein oder zwei Gastsemester an einer europäischen Partneruniversität zu verbringen. Fünf Jahre zuvor waren es erst 115000 , vor zehn Jahren knapp 80 000. Statistiken sind dokumentiert unter http://ec.europa.eu/education/ programmes/llp/erasmus/stat_en.html (zuletzt abgerufen am 25.11.2009). 
stituten an deutschen Universitäten etabliert. Doch werden freilich nicht allein in den relativ wenigen dezidiert internationalen Studiengängen und von der Handvoll Professoren mit explizit internationaler Widmung Kurse mit inter- oder transnationalen Inhalten angeboten.

Wie viele diesbezügliche Vorlesungen, Seminare und Übungen an den deutschsprachigen kommunikations- und medienwissenschaftlichen Instituten genau abgehalten werden, suchten wir mittels einer Lehrangebotsanalyse zu ermitteln. Die Anlage der Erhebung beruht auf einer Durchsicht der online zur Verfügung stehenden (wo immer zugänglich kommentierten) Vorlesungsverzeichnisse für das Sommersemester 2007 und das Wintersemester 2007/08 aller auf der DGPuK-Homepage verlinkten deutschen Institute (www.dgpuk.de - dort unter "Service“/ Institute im deutschsprachigen Raum“). Von den 42 aufgeführten Instituten (Stand März 2008) waren im untersuchten Sommersemester wie im Wintersemester 2007/08 je 40 Vorlesungsverzeichnisse verfügbar. ${ }^{3}$ In einem ersten Schritt wurden alle Lehrveranstaltungen mit erkennbarem internationalen oder interkulturellen Schwerpunkt erfasst. ${ }^{4}$ Ziel war dabei nicht, die Curricula an sich $\mathrm{zu}$ evaluieren, sondern eine Bestandsaufnahme der Kurse zu liefern.

Insgesamt wurden 145 Kurse an den 40 von uns untersuchten Instituten angeboten, davon 67 im Sommer- und 78 im Wintersemester. Im Durchschnitt ergibt das über alle Institute im Jahr 3,6 Kurse (und damit deutlich weniger als zwei Veranstaltungen pro Institut und Semester). Allerdings ist eine sehr ungleiche Verteilung festzustellen (vgl. Tabelle 1): Neun Institute bieten keinen einzigen Kurs in diesem

3 Genaue Vorlesungsverzeichnisse konnten vom Studiengängen Onlinejournalismus und Wissenschaftsjournalismus der Hochschule Darmstadt und von der Fachhochschule Oldenburg-Ostfriesland-Wilhelmshaven nicht ermittelt werden.

4 Gezählt wurden alle Vorlesungen, Seminare, Übungen etc., die (sofern aus Veranstaltungstiteln oder den Kommentaren in den kommentierten Vorlesungsverzeichnissen erkennbar) entweder einen Überblick oder Einblick in inter- oder transnationale respektive transkulturelle Fragestellungen gaben (also etwa Einführungskurse in internationale Mediensysteme) oder komparatistisch angelegt waren (also beispielsweise Mediensysteme oder Medienentwicklungen in zwei oder mehr Ländern vergleichend in den Blick nahmen - z. B. Kurse über lokale Nachrichtenangebote in verschiedenen europäischen Staaten, zu Mediensystemen in der arabischen Welt oder zur Entwicklung einer „europäischen Öffentlichkeit“), oder Ereignisse, Entwicklungen, Phänomene o. ä. in wenigstens einem anderen Staat zum Gegenstand hatten (z.B. Seminare über das Mediensystem Frankreichs, über Lokalradios in Brasilien oder über aktuelle Kinoentwicklungen in Hollywood). Internationalität wurde so in einem weitem Sinne verstanden. 
Themenbereich an, zwölf weitere höchstens zwei Kurse; sechs Institute offerieren hingegen neun oder mehr einschlägige Lehrangebote. Spitzenreiter ist das Institut für Kommunikations- und Medienwissenschaft der Universität Leipzig mit zwölf international ausgerichteten Lehrveranstaltungen im akademischen Jahr 2007/08, dicht gefolgt vom Institut für Kommunikationswissenschaft und Medienforschung (IfKW) der Universität München mit elf Kursen. Mindestens teilweise erklärbar ist das allein mit der Größe dieser Einrichtungen, beide Institute bieten in jedem Jahr insgesamt mehr als 200 Lehrveranstaltungen $^{5}$ an (so dass international ausgerichtete Kurse kaum fünf Prozent des Gesamtangebots ausmachen). Je zehn Kurse gibt es an der Sektion für Publizistik und Kommunikation der Ruhr-Universität Bochum und im Studiengang Kommunikationswissenschaft der Universität Erfurt, was wohl auch damit zu erklären ist, dass an beiden Einrichtung eine Professur mit internationaler Widmung eingerichtet ist (in Bochum besetzt mit Barbara Thomaß, in Erfurt mit Kai Hafez). Neun einschlägige Lehrveranstaltungen bieten im untersuchten Jahr die (wiederum sehr großen) Institute an den Universitäten Hamburg und Dortmund.

\begin{tabular}{|c|c|c|c|}
\hline Gesamtzahl der Kurse pro Institut & $\begin{array}{c}\text { Sommer- } \\
\text { semester } \\
2007\end{array}$ & $\begin{array}{c}\text { Winter- } \\
\text { semester } \\
2007 / 2008\end{array}$ & $\begin{array}{c}\text { Sommersemester 2007 } \\
\text { + Wintersemester } \\
2007 / 08\end{array}$ \\
\hline Kein Kurs & 15 & 13 & 9 \\
\hline 1 Kurs & 8 & 8 & 6 \\
\hline 2 Kurse & 7 & 6 & 6 \\
\hline 3 Kurse & 4 & 3 & 1 \\
\hline 4 Kurse & 1 & 4 & 6 \\
\hline 5 Kurse & 1 & 3 & - \\
\hline 6 Kurse & 4 & 2 & 2 \\
\hline 7 Kurse & - & 1 & 1 \\
\hline 8 Kurse & - & - & 2 \\
\hline 9 Kurse & - & - & 1 \\
\hline 10 Kurse & - & - & 1 \\
\hline 11 Kurse & - & - & 145 \\
\hline 12 Kurse & - & - & \\
\hline Gesamtzahl der Kurse & 67 & 78 & 3 \\
\hline
\end{tabular}

Tab. 1: Verteilung der Kursangebote im Bereich „internationale und interkulturelle Kommunikation"

5 Ausweislich der Vorlesungsverzeichnisse wurden am Münchner Institut 95 Kurse im Sommersemester 2007 und 120 im Wintersemester 2007/08 angeboten, im akademischen Jahr 2007/08 also insgesamt 215 Lehrveranstaltungen. Am Leipziger Institut waren etwa eben so viele Kurse angekündigt. 
Aus dieser Erhebung können wir einige Trendbeobachtungen vornehmen: Nur an wenigen Instituten gibt es ganze Module mit internationalem Bezug (so z. B. an den kommunikations- oder medienwissenschaftlichen Instituten der Universitäten in Dortmund, Düsseldorf, Erfurt, Hamburg und Weimar und an den spezifischen Studiengängen etwa der Fachhochschulen in Bremen, Hannover, Iserlohn und Stuttgart). An vielen Instituten sind hingegen international ausgerichtete Kurse gar nicht fest im Curriculum verankert, sondern werden (wenn überhaupt) nur als Spezifizierungen im Rahmen allgemeinerer Labels (so zum Beispiel ein Kurs über die "challenge of international corporate communications" unter dem Label „Marketing“ oder ein Seminar zur „Internationalen Krisen- und Konfliktberichterstattung“ im Modul „Theorie und Praxis des Journalismus“, beide in München) oder nur als zusätzliche Kurse angeboten. Oft hängt die "Internationalität" stark an Einzelpersonen (wie eben an Kai Hafez in Erfurt und Barbara Thomaß in Bochum oder an Christina Holtz-Bacha in Nürnberg, an Gerhard Piskol und Marcel Machill in Leipzig, an Hartmut Weßler in Mannheim, an Martin Löffelholz in Ilmenau oder an Andreas Hepp in Bremen). Dementsprechend wirken internationale Angebote teilweise nur wie „Steckenpferde“, die von einigen Dozenten - sehr verdienstvoll - „geritten“ werden. So etwa, wenn Gerhard Piskol in Leipzig Semester für Semester Hauptseminare anbietet $\mathrm{zu}$ „Mediensysteme im Vergleich: Russland - Polen Tschechien“ (Sommersemester 2007), zum "Mediensystem der USA“ (Sommersemester 2007 und Wintersemester 2007/08) oder zum „Mediensystem der Republik Frankreich“ (Wintersemester 2007/08), wenn Gunter Reus am Institut für Journalistik und Kommunikationsforschung der Hochschule für Musik und Theater Hannover eine Summer-School in Sibirien anbietet (Sommersemester 2007) oder wenn sich Hans Bohrmann in Dortmund "typischen Ausformungen von Medien und Journalismus in Europa" annimmt (Wintersemester 2007/08) - jeweils ohne dass derartige Lehrangebote explizit im Curriculum vorgesehen wären.

Viele der einschlägigen Lehrveranstaltungen werden von Lehrbeauftragten abgehalten. Nicht wenige haben einen sehr speziellen Fokus - z. B. Seminarangebote zur „Qualitativen Forschung zur Mediennutzung in Südkorea“ (von Klemens Schwitzer an der Universität Düsseldorf), zur „Political-Correctness-Debatte in Deutschland und Amerika" (von Andreas Jahn-Sudmann an der Universität Göttingen) oder über „Deutsche TV-Serien im internationalen Vergleich“ (von Tanja Weber an der Universität Köln) - und sind erkennbar weniger als Einführungen in diesem Forschungsbereich gedacht. 
Relativ am häufigsten sind dennoch Einführungsveranstaltungen zum Überblick über Mediensysteme (insgesamt finden sich in den 40 untersuchten Instituten in den zwei Semestern 22 derartige Angebote). Weitere Schwerpunkte in den Lehrangeboten lassen sich in den Bereichen „Medien und Europa (Europäische Öffentlichkeit / EU-Medienpolitik)“ mit 13 einschlägigen Kursen und „Kriegs- und Krisenkommunikation oder -berichterstattung" mit zehn Seminaren und Übungen feststellen.

Selten sind hingegen (noch) englischsprachige Lehrveranstaltungen: Nur zwölf der 145 insgesamt angebotenen ,internationalen“ Kurse fanden (soweit aus den Vorlesungsverzeichnissen erkennbar) in der Lingua Franca internationaler Kommunikation statt - und vier davon allein im englischsprachigen Euro-Mundus Masterstudiengang "Journalism and Media within Globalisation" der Universität Hamburg.

Die Kursformen lassen sich anhand der online verfügbaren Vorlesungsverzeichnisse nur vage ermitteln, schon da es an verschiedenen Instituten oft unterschiedliche Bezeichnungen gibt. Zusammenfassend kann man aber feststellen, dass Seminare im Bereich der „internationalen" Lehre an deutschen Instituten bei weiten überwiegen; auch Übungen finden sich noch recht häufig, Vorlesungen hingegen nur wenige (vgl. Tabelle 2).

\begin{tabular}{|l|r|}
\hline Seminare (inklusive Proseminare, Projektseminare u.ä.) & 83 \\
\hline Hauptseminare & 14 \\
\hline Übungen (inklusive Paxisseminare u.ä.) & 25 \\
\hline Vorlesungen & 5 \\
\hline Nicht zuordenbar & 18 \\
\hline Gesamt & 145 \\
\hline
\end{tabular}

Tab. 2: Typen der Lehrveranstaltungen im Bereich „internationale und interkulturelle Kommunikation"

Insgesamt kann man aus den (unterschiedlich umfänglich dargebotenen) Informationen über die einzelnen Lehrveranstaltungen in den Vorlesungsverzeichnissen zwar einiges über die Themen erfahren, weit weniger aber über konkrete Inhalte wie auch über methodische Zugänge - und kaum etwas über die detaillierte Konzeption der Lehrveranstaltungen, also etwa über Aufbau, Ablauf und didaktisches Konzept. An einem Beispiel soll nun gezeigt werden, wie es möglich sein kann, Theorie und Praxis - Vermittlung von Grundlagenkenntnissen, Austausch mit Wissenschaftlern und Medienschaffenden sowie Einübung handwerklicher Fertigkeiten - in einer Kursform zu kombinieren. 


\section{Seminarreihe „Bildkorrekturen"}

Im Zentrum von „Bildkorrekturen“ steht eine Konferenz, die seit 2002 von Internationale Weiterbildung und Entwicklung gGmbH (InWEnt) ${ }^{6}$ in Kooperation mit der Deutschen Journalistenschule (DJS), den Nachwuchsjournalisten in Bayern (NJB) und dem IfKW sowie seit 2007 auch der Ausbildungsredaktion des Bayerischen Rundfunks veranstaltet wird. Sie findet jährlich in der ersten Dezemberhälfte im Tagungsgebäude von InWEnt in Feldafing statt und führt jedes mal rund achtzig Journalisten, Wissenschaftler, Experten aus Politik, Entwicklungszusammenarbeit und von NGOs zusammen - und auch Studierende. Ein Kernanliegen ist der Informations- und Meinungsaustausch über Anforderungen an die und Probleme der "Nord-Süd-Berichterstattung“. An sich jährlich ändernden regionalen Schwerpunkten und inhaltlichen Fallbeispielen sollen Defizite in der Berichterstattung und die Zwänge für die Berichterstatter aufgezeigt und diskutiert werden. Alle Teilnehmer sollen für (mehr) Qualität in der (Auslands-)Berichterstattung sensibilisiert werden. Als Abschluss sollen gemeinsam alternative Zugänge und Lösungsvorschlägen erarbeitet werden.

Um allen eine sprachlich möglichst „barrierefreie“ Mitwirkung zu ermöglichen, wird die bilinguale Tagung simultan auf deutsch, englisch und teilweise auch französisch übersetzt. Der dreitägige Workshop bietet Keynote-Vorträge von Wissenschaftlern wie Hans J. Kleinsteuber und Johan Galtung, Darstellungen der Problemlagen von Experten aus Nord und Süd sowie Arbeitsberichte von Journalisten aus Deutschland (Korrespondenten) und vor allem auch aus dem „Süden“ selbst, also aus Staaten und Regionen, die im Mittelpunkt der Diskussionen stehen. Abstrakte Probleme werden an konkreten Beispielen besprochen. So stand „Bildkorrekturen“ 2004 unter dem Motto „Nur Hunger und Armut? Berichterstattung über das tägliche Leben im Süden“; im Fokus stand das Alltagsleben in Brasilien, dem Iran und Nigeria. 2005 war man „Der Desertifikation auf der Spur. Wie der Entwicklung der Boden entzogen wird“ - mit Fallbeispielen aus Brasilien sowie aus Indien, Mali und Jemen. 2007 war „Die Rolle von Medien in Krisengebieten" am Beispiel von Afghanistan, Kongo und dem Kosovo Thema, und am letzten Novemberwochenende 2008

6 InWEnt ist ein Träger der deutschen Entwicklungszusammenarbeit und entstand 2002 als Zusammenschluss der Deutschen Stiftung für internationale Entwicklung und der Carl-Duisberg-Gesellschaft. Hauptgesellschafter ist die Bundesregierung, vertreten durch das Bundesministerium für wirtschaftliche Zusammenarbeit und Entwicklung. Projektleiter der Reihe „Bildkorrekturen“ bei InWEnt ist F. Kayode Salau. 
befasste man sich anlässlich der Olympischen Spiele in Peking (retrospektiv) und der Fußball-Weltmeisterschaft in Südafrika (prospektiv) mit dem Gegenstandsbereich „Sport und Entwicklung“.

Die Studierenden - pro Jahr 30 Studentinnen und Studenten des Diplomstudiengangs Journalismus (seit 2008 Masterstudiengang Journalismus) am IfKW - haben dabei eine tragende Rolle. ${ }^{7}$ Sie erarbeiten im Vorfeld im Rahmen eines Seminars (das im Curriculum unter dem Label „Theorie und Praxis des Journalismus“ gefasst ist und bisher vom universitären Projektkoordinator Markus Behmer geleitet wurde) am IfKW in Kooperation mit der Deutschen Journalistenschule den Forschungsstand, führen eine Inhaltsanalyse der spezifischen Berichterstattung durch und entwickeln Leitfragen für den Workshop. Während der Tagung selbst bringen sie sich in die Diskussionen ein, übernehmen kurze Statements und führen Interviews. In der Nachbereitung der Tagung erarbeiten sie dann (anstelle einer wissenschaftlichen Seminararbeit) eine Dokumentation, die schließlich auch gedruckt und in einer Auflage von rund 3000 Stïck verbreitet wird (z.B. Behmer/ Salau/Wimmer 2003; InWEnt 2005, InWEnt 2006, InWEnt 2009).

Mit diesem Ablauf sollen vielfältige spezifische journalistische Kompetenzen vermittelt werden (vgl. Weischenberg 1990): Sachkompetenz wird mit dem Faktenwissen zum jeweiligen Schwerpunkt erworben. Eine größere Fachkompetenz entsteht durch das im Rahmen des Workshops diskursiv angeeignete Wissen über die medialen $\mathrm{Zu}$ sammenhänge, Zwänge und Möglichkeiten im Bereich der internationalen und interkulturellen Kommunikation. Die Begegnung und der Austausch mit den in- wie ausländischen Fachreferenten erhöht die Vermittlungskompetenz der Studierenden - z.B. in einem tieferen, anschaulich erworbenen Wissen um Recherchemöglichkeiten, Anforderungen an Korrespondenten, Erwartungen an die Medien von Seiten Betroffener etc. Der im Workshop stattfindende Dialog erweitert auch die Reflexionskompetenz. So schärft er das Rollenverständnis der angehenden Journalisten und schafft Bewusstsein für ethische Implikationen, für spezifische Funktionen der Medien im „Nord-SüdDialog" wie auch für Möglichkeiten und Grenzen einer kritischen Berichterstattung etwa in Staaten mit eingeschränkter Pressefreiheit. Technische Kompetenz wird schließlich durch die Anwendung des Erlernten z.B. während der Erstellung der Dokumentation gefördert.

7 Auch in anderen Studiengängen des IfKW finden tagungsbegleitende Seminare statt. So wurde 2003 unter reger Beteiligung der Magisterstudierenden eine Inhaltsanalyse zur Berichterstattung über Afrika durchgeführt (Wimmer 2003). 


\section{Resümee}

Anhand unserer knappen Bemerkungen zu allgemeinen Entwicklungen im Bereich der Lehre mit internationalen und interkulturellen Inhalten an deutschen Hochschulen wie auch insbesondere unserer Auszählung der konkreten Lehrangebote sollte deutlich geworden sein, dass die Bedeutung internationaler Themen auch für die Lehre weithin anerkannt ist und dass wenigstens an einigen Universitäten ein relativ differenziertes Lehrangebot vorliegt. Auch sind zuletzt einige spezifische, international ausgerichtete Masterstudiengänge entstanden. Dieser an sich positive Prozess der Ausweitung und zunehmenden Differenzierung findet jedoch nicht im gleichen Maße wie in anderen Bereichen der universitären kommunikations- und medienwissenschaftlichen Lehre (wie etwa in der Methodenausbildung, im Kommunikationsmanagement etc.) statt. Die bloße Anzahl an (vor allem) Seminaren und Übungen sollte auch nicht darüber hinwegtäuschen, dass Gegenstände der internationalen Forschung vielfach noch kaum fest in den Curricula der meisten Studiengänge verankert sind und dass an vielen Universitäten und Fachhochschulen (noch) nur wenige, teilweise gar keine einschlägigen Kurse angeboten werden. Auch scheint kaum Einigkeit darüber zu bestehen, welche Inhalte konkret zu vermitteln sind - eine Art „Mindestanforderungskatalog“ für "internationale“ Lehrveranstaltungen ist jedenfalls nicht erkennbar. An vielen Standorten werden oft eigene Spezifika gepflegt - mit nicht selten eher zufällig wirkenden Schwerpunkten und ohne dass eine echte Nachhaltigkeit in der Vermittlung gewisser Standards zu erkennen wäre.

Abschließen möchten wir daher mit einem Plädoyer für mehr Lehre in diesem Bereich. Auch sollte es im Sinne einer Institutionalisierung mehr Austausch über die Lehre geben. Das soll keine Standardisierung der Lehre implizieren; vielmehr sollte der Erfahrungsaustausch im Vordergrund stehen und allmählich ein Netz gewoben werden, mittels dessen die Bedeutung des Gegenstandsbereichs Internationale und Interkulturelle Kommunikation klarer akzentuiert werden könnte. Dieser Erfahrungsaustausch könnte auch konkret über bestimmte Vermittlungsformen geführt werden, um - im Sinne einer „best practice" - Lehrveranstaltungen gemeinsam und gezielt weiterentwickeln zu können. Die (wenigen) Professuren mit spezifischer Widmung in diesem Bereich könnten hier als „Knotenpunkte“ fungieren, und das sich im Aufbau befindliche Netzwerk „Interkulturelle und Internationale Kommunikation'“ innerhalb der DGPuK könnte den institutionellen Rahmen für weiterführende Diskussionen bilden. 
Sinnvoll wäre sicher auch eine Ausweitung englischsprachiger Lehrangebote gerade bei internationalen Themensetzungen und eine verstärkte Kooperation mit ausländischen Kollegen (die so auch erleichtert würde) bis hin zur Erarbeitung gemeinsamer Kursprojekte oder der Einbindung von internationalen Gästen in eigene Lehrveranstaltungen. Gelegentliche Blockseminare von Kollegen aus europäischen Partneruniversitäten lassen sich zum Beispiel ohne sehr großen Aufwand mittels der „Staff Mobility“-Möglichkeit organisieren, die das Erasmus-Programm bietet.

Allerdings ist trotzdem fraglich, inwieweit dieser von uns postulierte Ausbau der Lehre administrativ wirklich gewollt ist respektive, ob es ausreichend Ressourcen dafür gibt. Inwieweit sind Fakultäten und Institute bereit, in diesen Bereich zu investieren? Sind dafür die nötigen Mittel vorhanden, gerade wenn man den oben skizzierten Empfehlungen des Wissenschaftsrates (Stipendien, Einbezug von Gastreferenten etc.) entsprechen möchte? Die von uns vorgestellte Seminarreihe „Bildkorrekturen“ würde ohne die große finanzielle Unterstützung seitens InWEnt nicht funktionieren.

Weiter bezieht sich die Ressourcenfrage nicht nur auf die Finanzierung; auch Fähigkeiten und Fertigkeiten sind angesprochen. Inwieweit stehen geeignete Mitarbeiter zu Verfügung, die Interesse an Lehre zu internationalen Themen haben und über die nötige Erfahrung sowie die inhaltliche Kompetenz verfügen? Damit verbunden ist auch die Frage des öffentlichen Interesses. Wir konnten immer wieder feststellen, dass Angebote in diesem Forschungsbereich sehr stark von den Studierenden nachgefragt werden und dass sie sich dann auch in den Lehrveranstaltung außergewöhnlich motiviert zeigen. Allerdings wird weiter entscheidend sein, inwieweit diese Seminarangebote und die darin vermittelten Kompetenzen letztlich auch auf dem Arbeitsmarkt honoriert werden? Diese Limitationen der international ausgerichteten Lehre brachte Hans-Henrik Holm schon vor einigen Jahren auf den Punkt (Holm 2002, S. 70): „The drawback of such programs is that they risk becoming isolated islands of internationalism that may work well for the teachers and students that participate, but have little overall effect on either the greater student body or perhaps even the institutions that participate in producing them."

Es bleibt abzuwarten, inwieweit diese Aussage - gerichtet zwar auf ein Journalismusprogramm der Entwicklungszusammenarbeit - sich nicht auch bei uns bewahrheitet. Fest steht für uns aber: Wenn in die Lehre im Bereich internationaler und interkultureller Lehre investiert wird, wenn an vielen Standorten (mehr) Kurse angeboten werden, wenn dadurch auch mehr studentische Abschlussarbeiten und letzt- 
lich auch mehr Forschungsarbeiten angeregt werden, die wiederum inhaltlich gewinnbringend in die Lehre zurückwirken können, dann wird die Relevanz des Themenbereichs immer mehr Menschen bewusst werden. Das kann dann wiederum auf die gesamte Gesellschaft zurïckwirken - und neue Chancen für gut ausgebildete Absolventen der Kommunikations- und Medienwissenschaft auch auf dem Arbeitsmarkt eröffnen. Wir sind überzeugt, dass es einen wachsenden Bedarf für sie in der zunehmend international agierenden Medienwirtschaft wie überhaupt in der (mindestens in der Nordhemisphäre) mehr und mehr global vernetzten Kommunikationsgesellschaft gibt.

\section{Literatur}

Baldasty, Gerald J. et al.( 2003): Has the dream stalled? In: Journalism Educator, 58. Jg., H.1, S. 7-25.

Behmer, Markus/Salau, F. Kayode/Wimmer, Jeffrey (Hg.) (2003): Nachrichten aus dem Weltdorf. „Dritte-Welt-Berichterstattung“ in deutschen Medien. Bonn.

Bilandzic, Helena/Lauf, Edmund/Hartmann, Thilo (2004): How to go international. DGPuK-Wegweiser zu internationalen Tagungen und Fachzeitschriften in der Kommunikationswissenschaft. München.

Esser, Frank/Pfetsch, Barbara (Hg.) (2003a): Politische Kommunikation im internationalen Vergleich. Grundlagen, Anwendungen, Perspektiven. Wiesbaden.

Esser, Frank/Pfetsch, Barbara (2003b): Politische Kommunikation im internationalen Vergleich: Neuorientierung in einer veränderten Welt. In: Dies. (Hg.): Politische Kommunikation im internationalen Vergleich. Grundlagen, Anwendungen, Perspektiven. Wiesbaden, S. 9-31.

Gurevitsch, Michael/Blumler, Jay G. (2003): Der Stand der vergleichenden politischen Kommunikationsforschung: Ein eigenständiges Feld formiert sich. In: Esser, Frank/Pfetsch, Barbara (Hg.): Politische Kommunikation im internationalen Vergleich. Grundlagen, Anwendungen, Perspektiven. Wiesbaden, S. 371-392.

Hafez, Kai (2002): International vergleichende Medienforschung: Eine unterentwickelte Forschungsdimension. In: Ders. (Hg.) Die Zukunft der internationalen Kommunikationswissenschaft in Deutschland. Hamburg, S. 59-94.

Hanitzsch, Dieter/Altmeppen, Klaus-Dieter (2007): Über das Vergleichen: Komparative Forschung in deutschen kommunikationswissenschaftlichen Fachzeitschriften 1948-2006. In: Medien \& Kommunikationswissenschaft, 55. Jg., H.2, S. 185-203. Hepp, Andreas ( 2006): Transkulturelle Kommunikation. Konstanz.

Hepp, Andreas/Krotz, Friedrich/Winter, Carsten (Hg.) (2005): Globalisierung der Medienkommunikation. Wiesbaden.

Hepp, Andreas/Löffelholz, Martin (Hg.) (2002): Grundlagentexte zur Transkulturellen Kommunikation. Konstanz. 
Holm, Hans-Henrik (2002): The forgotten globalization of journalism education. In: Journalism \& Mass Communication Educator, 57. Jg., H.1, S. 62-71.

InWEnt (Hg.) (2005): Bildkorrekturen. Nur Hunger und Armut? Berichterstattung über das tägliche Leben im Süden. Bonn.

InWEnt (Hg.) (2006): Bildkorrekturen. Der Desertifikation auf der Spur. Wie der Entwicklung der Boden entzogen wird. Bonn.

InWEnt (Hg.) (2009): Bildkorrekturen. Die Rolle der Medien in Krisengebieten. Bonn.

Kleinsteuber, Hans J. (2002): Mediensysteme im Vergleich. Ein Überblick. In: Hafez, Kai (Hg.): Die Zukunft der internationalen Kommunikationswissenschaft in Deutschland. Hamburg, S. 39-58.

Koivisto, Juha/ Thomas, Peter (2007): Mapping Communication and Media Research: Germany. Communication Research Center, University of Helsinki, Department of Communication, Research Reports 6/2007. In http:// http://www.valt.helsinki.fi/ blogs/crc/ReportGermany.pdf.

Kunzcik, Michael (2002): Medien und Entwicklungsländer. Versuch einer Bilanz. In: Hafez, Kai (Hg.): Die Zukunft der internationalen Kommunikationswissenschaft in Deutschland. Hamburg, S. 95-112.

Livingstone, Sonia (2003): On Challenges of Cross-National Comparative Media Research. In: European Journal of Communication, 18. Jg., H.4, S. 477-500.

Luyendijk, Joris (2007): Wie im echten Leben. Berlin.

Melischek, Gabriele/Seethaler, Josef/Wilke, Jürgen (Hg.) (2008): Medien und Kommunikationsforschung im Vergleich: Grundlagen, Gegenstandsbereiche, Verfahrensweisen. Wiesbaden.

Seethaler, Joseph (2006): Entwicklung und Stand der kommunikationswissenschaftlichen Forschung zur europäischen Öffentlichkeit. Eine Analyse der Beiträge in vier europäischen Fachzeitschriften 1989 bis 2004. In: Langenbucher, Wolfgang R./Latzer, Michael (Hg.): Europäische Öffentlichkeit und medialer Wandel. Eine transdisziplinäre Perspektive. Wiesbaden, S. 244-260

Thomaß, Barbara (Hg.) (2007): Mediensysteme im internationalen Vergleich. Konstanz.

Unesco (2007): Model curricula for journalism education for developing countries \& emerging democracies. Paris.

Weischenberg, Siegfried (1990): Das „Prinzip Echternach“. Zur Einführung in das Thema „Journalismus und Kompetenz“. In: Ders. (Hg.): Journalismus \& Kompetenz. Qualifizierung und Rekrutierung für Medienberufe. Opladen, S. 11-41.

Wimmer, Jeffrey (2003). Das Ende der „Dritten Welt.“ Ein Vergleich der Berichterstattung über Afrika in der deutschen Presse 1991 und 2001. In: Communicatio Socialis, 36. Jg, H. 4, S. 337-352.

Wissenschaftsrat (2007): Empfehlungen des Wissenschaftsrats zur Weiterentwicklung der Kommunikation- und Medienwissenschaften in Deutschland. In: http:// www.wissenschaftsrat.de/texte/7901-07.pdf. 